\title{
Senkyunolide I Protects Against Sepsis-associated Encephalopathy by Attenuating Sleep Deprivation in a Murine Model of Cecal Ligation and Puncture
}

Jian Xie

Changhai Hospital

Zhen-zhen Zhao

Changhai Hospital

Peng Li

Changhai Hospital

Cheng-long Zhu

Changhai Hospital

Yu Guo

Changhai Hospital

Jun Wang

Changhai Hospital

Xiao-ming Deng

Changhai Hospital

Jia-feng Wang ( $\nabla_{\text {jfwang@smmu.edu.cn ) }}$

Changhai Hospital

Research

Keywords: Sepsis associated encephalopathy, Senkyunolide I, Sleep deprivation, Inflammatory response

Posted Date: December 3rd, 2020

DOI: https://doi.org/10.21203/rs.3.rs-117431/v1

License: (1) (1) This work is licensed under a Creative Commons Attribution 4.0 International License.

Read Full License 


\section{Abstract}

Background: Sepsis may lead to sleep deprivation, which will promote the development of neuroinflammation and mediate the progression of sepsis associated encephalopathy (SAE).

Senkyunolide I, an active component derived from an herb medicine, has been shown to provide sedative effect to improve sleep. But its role in sepsis is unclear. The present study was performed to investigate whether Senkyunolide I protected against SAE in a murine model of cecal ligation and puncture (CLP).

Methods: The male C57BL/ 6 mice were used to investigate the effects of Senkyunolide I on SAE. The related protein of the sleep deprivation and inflammatory signaling pathway was detected by western blot. The activation of microglia and the neuronal apoptosis were separately detected by immunofluorescence staining and TUNEL staining.

Results: Here, we showed that Senkyunolide I treatment improved the 7-day survival rate and reduced the excessive release of cytokines including TNF- $\alpha$, IL- 6 and IL-1 $\beta$. A fear conditioning test was performed and the result showed that Senkyunolide I attenuated CLP-induced cognitive dysfunction. Senkyunolide I treatment also decreased the phosphorylation levels of inflammatory signaling proteins, including $p$-ERK, p-JNK, p-P38, p-P65, and the level of inflammatory cytokines, including TNF-a, IL-6 and IL-1 $\beta$, in the hippocampus homogenate. The sleep deprivation was attenuated by Senkyunolide I administration, as demonstrated by the modification of the BDNF and c-FOS expression. When sleep deprivation was induced manually, the protective effect of Senkyunolide I against inflammatory responses and cognitive dysfunction was reversed.

Conclusion: Our data demonstrated that Senkyunolide I could protect against sepsis-associated encephalopathy in a murine model of sepsis via relieving sleep deprivation.

\section{Introduction}

Sepsis, termed as life-threatening organ dysfunction induced by polymicrobial infection, is a leading cause of death in the intensive care unit (ICU) $(1,2)$. Sepsis may lead to diffuse brain dysfunction in the absence of central nervous system infection, which is also called sepsis-associated encephalopathy (SAE) with a prevalence up to $70 \%$ among patients with severe systemic infection $(3,4)$. The clinical manifestations of SAE vary greatly, from compromise in attention and orientation to delirium and even coma(5). Neuronal apoptosis, microcirculatory dysfunction and mitochondrial dysfunction has been implicated in SAE (6-8). The local production of pro-inflammatory mediators may resulting in impairment of central nervous system, and thus, microglia activation and the release of inflammatory cytokines, such as TNF-a and IL-1 $\beta$, play a central role in the development of $\operatorname{SAE}(9,10)$.

It has been observed that sleep quality may be impaired in septic rats (11). In human patients, sepsis might also induce changes in electroencephalogram (EEG) rhythm during sepsis (12). Therefore, sleep deprivation may be more frequent than we expected during sepsis. It has been reported that sleep deprivation can lead to release of inflammatory factor, cachexia and even death in sepsis (13-15). 
Therefore, improving sleep quality may be helpful to reduce the level of inflammation and improve the prognosis of sepsis.

Senkyunolide I, which is one of the effective components in a herb medicine named Ligusticum Chuangxiong hort, has been widely studied because of its analgesic and sleep-improving effects (1618). Peng et al. (17) reported that Senkyunolide I provided a sedative effect and was synergetic with pentobarbital in prolonging the sleeping time in mice. In another study performed by Guo et al. (19), Senkyunolide I was observed to be able to decrease the sleep latency in mice. Therefore, we speculated that Senkyunolide I might improve sleep quality and thereby protect against sepsis-induced inflammatory response and brain dysfunction induced by sleep disorder. The present study was performed to investigate the role of Senkyunolide I in the inflammatory level, sleep deprivation and brain impairment with a murine model of cecal ligation and puncture (CLP).

\section{Materials And Methods}

\section{Animals}

C57BL/6J mice (male, 8-10 weeks old) were obtained from GemPharmatech Experimental Animal Corporation (Nanjing, China). All animals were maintained in a room with proper conditions $\left(21^{\circ} \mathrm{C}-23^{\circ} \mathrm{C}\right.$, $40 \%-60 \%$ humidity) and $12 \mathrm{~h}$ light/dark cycle. Mice had access to food and water freely. The protocol of the animal study was approved by the ethics committee for animal research of Changhai Hospital, and conformed to the relevant rules and regulations.

\section{CLP model}

The CLP procedure was performed as previously reported. Generally, mice were anesthetized with 2-3\% sevoflurane and subcutaneous butorphanol $(1 \mathrm{mg} / \mathrm{kg})$ was used for analgesia. After skin disinfection, a middle abdominal incision was made and the cecum was exposed. In CLP mice, the cecum was ligated at the half from the root and punctured through with a $22 \mathrm{G}$ needle. Some cecal content was squeezed out and the cecum was returned to the abdominal cavity. In the sham-operated mice, the cecum was exposed without any treatment. Then the skin was closed in one layer and $1 \mathrm{ml}$ saline was injected subcutaneously for fluid resuscitation.

For most of the study, mice were euthanized at $24 \mathrm{~h}$ after operation for sample harvesting, including the hippocampus, cerebral cortex and the whole blood obtained by heart puncture. For survival analysis, the mortality was observed for seven consecutive days.

\section{Fear conditioning (FC) test}

A fear conditioning device (XR-XZ301, Xinruan Information Technology, Shanghai, China) was used to test the conditional memory as reported by $\mathrm{Ji}$ et al (20). Briefly, at the training stage, mice were exposed in the chamber for $3 \mathrm{~min}$, followed by stimulation with a tone $(30 \mathrm{~s}, 65 \mathrm{~dB}, 3 \mathrm{kHz})$ and a foot electrical stimulus ( 3 s $0.75 \mathrm{~mA}$ ). The contextual and tone fear conditioning test was carried $24 \mathrm{~h}$ later. Freezing 
time to context and tone during the 3-minutes period was recorded respectively. The freezing behavior is defined as no visible movement other than respiration.

\section{Sleep deprivation (SD) model}

A new sleep deprivation device consisting of a cylinder and an inbuilt interfering rod was used for mice (XR-XS108, Xinruan Information Technology, Shanghai, China). The mice were allowed to roam freely in their cylinder, without limitation to food and water. In order to avoid sleeping, interfering rod rotates consistently at a constant speed $(15 \mathrm{rpm})$ in the chamber to accomplish the sleep deprivation process. The process of sleep deprivation began from the time of fully recovery from anesthesia and lasted for $24 \mathrm{~h}$.

\section{Drug treatment and groups}

Senkyunolide I (C12H1604, CAS, 94596-28-8, purity $\geq 98 \%$ ) was purchased from Zheyan Bio-technology (Shanghai, China). Senkyunolide I was dissolved in dimethyl sulfoxide (DMSO, at a concentration of less than $5 \%$ ) for animal experiments and injected intraperitoneally $(36 \mathrm{mg} / \mathrm{kg})$. The dose was determined in accordance to previous studies (21).

For the pharmacological experiments, the mice were allocated equally into four groups, including Sham + DMSO group, Sham + SEl group, CLP + DMSO group and CLP + SEl group. The mice were administered with DMSO solution or Senkyunolide I solution dissolved in DMSO according to the grouping. For survival analysis, 96 mice were used with 24 mice in each group.

\section{Immunofluorescence assay}

The hippocampal tissues were harvested and cut into paraffin sections which were used for the immunofluorescence assay. Paraffin-embedded hippocampal tissue was cut into a thickness of $10 \mu \mathrm{m}$ on glass slides, which were then blocked with $10 \%$ donkey serum for 60 minutes and incubated with primary antibody (mouse anti-IBA-1, Proteintech, Illinois, USA) at $4{ }^{\circ} \mathrm{C}$ overnight. Secondary antibody was incubated for $1.5 \mathrm{~h}$ in room conditions. The nuclear staining was labeled with 4',6-diamidino-2phenylindole (DAPI). Finally, average IBA-1 number of positive staining in three fields under fluorescence microscope (Leica, Wetzlar, Germany) and five sections of each group was analyzed.

\section{TUNEL staining}

To investigate whether Senkyunolide I treatment could reduce the apoptotic rates of the neurons, apoptosis in hippocampus was detected by TUNEL assay (Roche, Basel, Switzerland). Briefly, the hippocampus tissues were harvested at $24 \mathrm{hrs}$ after operation and fixed in $4 \%$ paraformaldehyde for section. When the sections were prepared, they were deparaffinized, hydrated and treated with proteinase $\mathrm{K}$. Then the sections were covered with $3 \% \mathrm{H}_{2} \mathrm{O}_{2}$ for 5 min at room temperature to block endogenous peroxidase and incubated with terminal deoxynucleotidyl transferase reaction mixture while TBS solution was alternatively applied in sham groups. Finally, we incubated the sections with streptavidin-HRP, 
diaminobenzidine and counterstained with hematine. The ratio of TUNEL-positive cells in 5 field in each section was used for the following statistical analysis.

\section{Enzyme-linked immunosorbent assay (ELISA)}

The concentrations of inflammatory mediators in samples of serum and hippocampus were quantified using ELISA kits (Thermo, Massachusetts, USA). The hippocampus was homogenized with cold normal saline and centrifuged at $10,000 \times \mathrm{g}$ for $10 \mathrm{~min}$ to obtain supernatants. Similarly, whole blood was centrifuged at $1500 \mathrm{rpm}$ for $10 \mathrm{~min}$ and the serum was collected. The procedures of ELISA assay were performed with the instructions provided by manufacturer (Invitrogen, Carlsbad, CA, USA). The results were read at $450 \mathrm{~nm}$ with a spectrophotometer (Synergy2, BioTek, USA).

\section{Western blot assay}

Proteins in hippocampal tissues were extracted with RIPA lysis buffer encompassing a protease inhibitor cocktail and the protein concentrations were measured with the BCA assay (Thermo, Massachusetts, USA). The denatured protein samples (30 $\mathrm{ug}$ per sample) were separated by $10 \%$ SDS-PAGE gel and transferred to polyvinylidene difluoride (PVDF) membranes (Merck, Darmstadt, Germany). The transferred membranes were blocked with $5 \%$ skimmed milk and then incubated with primary antibody at $4^{\circ} \mathrm{C}$ overnight. The HRP-conjugated secondary antibody was added to incubate the washed membrane for $3 \mathrm{~h}$ at room temperature. Finally, the membranes were visualized with ECL reagent (Thermo, Massachusetts, USA). The protein bands were analyzed by using Image J software (National Institutes of Health, Maryland, USA). The primary antibodies included p-ERK (1:1000, CST, Boston, USA), ERK (1:1000, CST, Boston, USA), p-JNK (1:1000, CST, Boston, USA), JNK (1:1000, CST, Boston, USA), p-P38(1:1000, CST, Boston, USA), P38(1:1000, CST, Boston, USA), p-P65(1:1000, CST, Boston, USA), P65(1:1000, CST, Boston, USA), BDNF (1:1000, Abcam, Massachusetts, USA), c-FOS (1:1000, CST, Boston, USA) and beta-actin (1:2000, Sigma, USA).

\section{Statistical analysis}

All data were statistically analyzed using the Graph Pad Prism 8.4 software (La Jolla, CA, USA). The data were presented as mean values \pm standard error of mean and compared using the one-way ANOVA. A P values $<0.05$ was considered as statistically significant.

\section{Results}

\section{Senkyunolide I improved survival rates and reduced systemic inflammation in septic mice}

In order to investigate the effects of Senkyunolide I on overall survival of mice, the CLP mice were administered with Senkyunolide I $(36 \mathrm{mg} / \mathrm{kg})$ and observed for 7 consecutive days. In the CLP + DMSO group, 13 of 24 mice survived on the seventh day (Fig. 1A), while Senkyunolide I administration could significantly improve the survival rates of CLP mice (4 of 24 mice survived, Fig. 1A, $p=0.0048$ ). 
ELISA was performed to analyze pro-inflammatory factors including TNF-a, IL-1 $1 \beta$, IL- 6 in serum. The serum levels of TNF- $a$ (Fig. 1B), IL-1 $\beta$ (Fig. 1C) and IL-6 (Fig. 1D) were significantly increased at $24 \mathrm{~h}$ after CLP operation. Compared with CLP + DMSO group, Senkyunolide I treatment remarkably decrease the concentrations of TNF- $a$, IL-1 $\beta$ and IL- 6 in plasma. These results indicated that Senkyunolide I administration after CLP operation was protective against sepsis challenge.

\section{Senkyunolide I attenuated the memory impairment in CLP mice}

Fear conditioning test was performed to assess the effects of Senkyunolide I on CLP-induced memory impairment. As shown in Fig. 2A and 2B, the time of freezing to context and the time of freezing to tone were significantly declined in the CLP + DMSO group than in their counterparts in the sham groups at $24 \mathrm{~h}$, whereas Senkyunolide I treatment attenuated the decline in the CLP + DMSO group. Thus, Senkyunolide I may have prevented CLP-induced memory impairment.

\section{Senkyunolide I alleviated apoptosis and suppressed the activation of microglia in the hippocampus region of sepsis mice}

TUNEL assay was performed to examine the neuroprotective effects of Senkyunolide I. It was shown that the rates of apoptotic cell were greatly increased under sepsis challenge, while Senkyunolide I treatment significantly inhibited neuronal apoptosis as shown in Fig. 3A and 3B. Microglia activation is a marker for neuroinflammation in SAE, which may be associated with neuronal injury. Therefore, we detected IBA-1, a marker of activated microglia, by immunofluorescence assay. The fluorescence intensity of IBA-1 was significantly higher in the CLP + DMSO group than in the sham groups, while Senkyunolide I treatment obviously reversed these changes (Fig. 4A, 4B), suggesting that Senkyunolide I may suppress microglial activation so as to inhibit neuroinflammation.

\section{Senkyunolide I inhibited the activation of inflammatory signaling pathway in the hippocampus}

MAPKs signaling pathway and NF-KB signaling pathway are involved in various inflammatory diseases and also contribute to the production of TNF-a, IL-1 $\beta$, IL-6, hence reducing neuronal plasticity and neurogenesis (22). These inflammatory signaling pathways were also demonstrated to be activated in the process of sepsis-associated encephalopathy $(23,24)$. Therefore, we detected the expression of inflammatory factors and the activation status of these signaling molecules in the hippocampus homogenate. The elevation of TNF-a (Fig. 5A), IL-1 $\beta$ (Fig. 5B) and IL-6 (Fig. 5C) in CLP mice were attenuated by the Senkyunolide I administration. Similarly, the phosphorylation levels of JNK, ERK, p38 and p65 were all enhanced in CLP mice but Senkyunolide I reduced the activation level significantly as shown in Fig. 5D-5H. 


\section{The anti-inflammatory effect of Senkyunolide I depended on the attenuation of sleep deprivation during sepsis}

Some studies have been confirmed that sleep deprivation is associated with immune dysfunction, inflammatory cytokine release, cachexia and other adverse events, thus, treatment targeting at sleep deprivation may be helpful in attenuating $\operatorname{SAE}(15,25)$. Since BDNF and c-FOS are markers related to sleep deprivation(26), we investigate whether Senkyunolide I can modulate the expression of these two proteins. The western blot assay revealed that expression level of BDNF was highly decreased while the cFOS level was significantly elevated in both of hippocampus and cerebral cortex in septic mice. However, Senkyunolide I treatment could reverse these alterations of BDNF and c-FOS induced by CLP (Fig. 6A-6F).

A cylinder device with an interfering rod was used to induce sleep deprivation to investigate whether attenuation of sleep deprivation was involved in the protective role of Senkyunolide I on activation of inflammatory responses. As revealed in Fig. 7A-7F, the effect of Senkyunolide I on expression of BDNF and c-FOS was reversed by induced sleep deprivation. Similar results were also observed in the plasma levels of the proinflammation cytokines that sleep deprivation diminish the protective role of Senkyunolide I against proinflammatory responses induced by CLP (Fig. 7G-7I). Furthermore, the fear conditioning test revealed that the freezing time to both of context and tone returned to the level of CLP mice even if Senkyunolide I was administered (Fig. 7J, 7K).

\section{Discussion}

In the present study, we investigated the therapeutic effect of Senkyunolide I in sepsis and SAE in a murine CLP model. Our data suggested that Senkyunolide I could improve the survival and inhibit systemic inflammation in CLP mice. Sepsis-induced memory impairment was attenuated by Senkyunolide I. Senkyunolide I reduced apoptosis, microglia activation, and the activation of MAPK and NF-KB signaling pathway in the hippocampus region. The alterations of the markers of sleep deprivation was attenuated by Senkyunolide I, including the downregulation of BDNF and upregulation of c-FOS. When the sleep deprivation was induced, the protective role of Senkyunolide I against systemic inflammation and memory impairment was reversed partially or completely.

Senkyunolide I, one of the main active ingredients of Ligusticum Chuangxiong hort, has good lipid solubility and can be absorbed quickly into the blood and cerebrospinal fluid. Senkyunolide I has been demonstrated to show anti-inflammation, anti-apoptosis and anti-oxidative effects in several studies of neural injury. Hu et al. $(21,27)$ reported that Senkyunolide I could alleviate stroke-induced neuroinflammation via suppressing the TLR4/NF-KB pathway and protect against cerebral reperfusion injury with its anti-apoptosis property. Another study showed that Senkyunolide I could reduce the sleep latency and exert a synergetic sleep-promoting effect (17). However, the role of Senkyunolide I on SAE is seldom reported. 
Inflammation plays a vital role in the pathogenesis of sepsis. The bacteria themselves and their metabolites can elicit an exorbitant inflammatory response and lead to profound release of inflammatory mediators, such as TNF- $a$, IL- 6 and IL-1 $\beta$. The over-released cytokines positively impact the evolvement of sepsis and even result in organ damage, finally lead to death (28). Our data demonstrated that the concentrations of proinflammatory cytokines were obviously elevated in CLP + DMSO group compared to those in the sham groups in both of serum and hippocampus homogenate. Senkyunolide I administration significantly reduced the levels of these cytokines and thereby improved the survival rate of septic mice.

Brain dysfunction induced by sepsis, which is also called SAE, is characterized by neuronal apoptosis and microbial activation $(29,30)$. Excessive metabolism of oxygen may be present during sepsis, leading to cytochrome c release, caspase-3 activation and ultimately apoptosis [22]. The number of TUNELpositive cells was significantly reduced by Senkyunolide I in hippocampus at $24 \mathrm{~h}$ after CLP operation, suggesting that Senkyunolide I attenuated apoptosis in the hippocampus. Furthermore, Senkyunolide I treatment suppressed the expression of IBA- 1 and release of IL-1 $\beta$ in hippocampus, which was a marker of microglial activation. Henry et al. (31) reported that systemic inflammation induced by peripheral inflammatory stimuli could lead to microglial activation and thus facilitate the release of IL-1 $\beta$ into the CNS. Another study showed that inhibition of microglial activation could decrease oxidative stress and cytokine levels in the hippocampus, which would improve cognitive behavior of septic mice(32). Further assays showed that activation of proinflammatory signaling pathways (NF-kB and MAPK) were also inhibited in the hippocampus. These data suggested that Senkyunolide I had neuroprotective effect against SAE.

The potential mechanism of the neuroprotective effect of Senkyunolide I remains unclear. It was interesting to notice that BDNF was reduced while c-FOS was elevated in septic mice, which indicated sleep deprivation was present in septic mice (26). Sleep deprivation might activate microglia and promote the release of inflammatory mediators (33). Other studies also reported that sleep deprivation would result in immune dysfunction and impair the capacity of host defense $(13,15,25)$. Therefore, it was not strange that Senkyunolide I attenuated sepsis-induced memory impairment when it reversed the alterations of the markers of sleep deprivation. The role of sleep deprivation seemed to be critical for Senkyunolide I, since induced sleep deprivation could reverse the protective effect of Senkyunolide I.

\section{Conclusion}

In conclusion, Senkyunolide I can alleviate sepsis-induced mortality and systemic inflammation. Senkyunolide I may also be protective against sepsis-associated encephalopathy as demonstrated by the apoptosis, microglial activation and the activation of MAPK and NF-KB signaling pathway. Sleep deprivation is attenuated by Senkyunolide I and may participate an important role of Senkyunolide I in protecting against sepsis- associated encephalopathy.

\section{Abbreviations}


SEl: Senkyunolide I; SAE: sepsis-associated encephalopathy; MAPK: Mitogen-activated protein kinase; NFKB: Nuclear factor -Kappa B; SDS-PAGE: Sodium dodecyl sulfate-polyacrylamide; PVDF: Polyvinylidene fluoride membrane; CLP: cecal ligation and puncture.

\section{Declarations}

\section{Authors' contributions}

Jian Xie, Zhen-zhen Zhao, and Peng Li contributed equally to the article. Jian Xie and Zhen-zhen Zhao performed the research and analyzed the data. Jian Xie and Peng Li wrote the major part of the manuscript. Jia-feng Wang and Xiao-ming Deng designed the research, ensured correct analysis of the data, and wrote the manuscript. Peng Li, Jian Xie, Cheng-long Zhu, Yu Guo, and Jun Wang assisted in the design of the research, oversaw the collection of the data, and contributed to the writing of the manuscript. All authors critically revised the manuscript and gave final approval of the manuscript.

\section{Funding}

This study was supported by the grants from Natural Science Foundation of China (82072147) and Shanghai Science and Technology Innovation Project (16411950300).

\section{Availability of data and materials}

The datasets during and/or analyzed during the current study are available from the corresponding author on reasonable request.

\section{Ethics approval and consent to participate}

All animal procedures performed in this study were performed strictly according to the international standards of animal care guidelines and have been approved by the Animal Care and Use Committee of Guangxi Medical University.

\section{Consent for publication}

Written informed consent for publication was obtained from all authors.

\section{Competing Interests}

The authors declare that there is no conflict of interest regarding the publication of this paper. 


\section{References}

1. Vincent J-L, Marshall JC, Ñamendys-Silva SA, François B, Martin-Loeches I, Lipman J, et al. Assessment of the worldwide burden of critical illness: the Intensive Care Over Nations (ICON) audit. The Lancet Respiratory Medicine. 2014;2(5):380-6.

2. Rhodes A, Evans LE, Alhazzani W, Levy MM, Antonelli M, Ferrer R, et al. Surviving Sepsis Campaign: International Guidelines for Management of Sepsis and Septic Shock: 2016. Intensive Care Med. 2017;43(3):304-77.

3. GB Y. Encephalopathy of infection and systemic inflammation. J Clin Neurophysiol. 2013;30:454-61.

4. TranDD, GroeneveldAB, van der MeulenJ. Age, chronic disease, sepsis, organ system failure, and mortality in a medical intensive care unit. Crit Care Med. 1990;18:474-9.

5. Gofton TE, Young GB. Sepsis-associated encephalopathy. Nat Rev Neurol. 2012;8(10):557-66.

6. Wang P, Hu Y, Yao D, Li Y. Omi/HtrA2 Regulates a Mitochondria-Dependent Apoptotic Pathway in a Murine Model of Septic Encephalopathy. Cell Physiol Biochem. 2018;49(6):2163-73.

7. Nicolas Adam¹, Stanislas Kandelman². Sepsis-induced brain dysfunction. Anti-Infect. 2013;11(2): 211-21.

8. Tighe D MR, Boghossian S. Multi-organ damage resulting from experimental faecal peritonitis. Clinical science (London, England: 1979). 1989;76(3):269-76.

9. M. Michels, L.G. Danielski, F. Dal-Pizzol, F. Petronilho. Neuroinflammation: microglial activation during sepsis. Curr Neurovasc Res. 2014 .11(3):262-70.

10. Moraes CA, Santos G, de Sampaio e Spohr TC, D'Avila JC, Lima FR, Benjamim CF, et al. Activated Microglia-Induced Deficits in Excitatory Synapses Through IL-1beta: Implications for Cognitive Impairment in Sepsis. Mol Neurobiol. 2015;52(1):653-63.

11. Baracchi F, Ingiosi AM, Raymond RM, Jr., Opp MR. Sepsis-induced alterations in sleep of rats. Am J Physiol Regul Integr Comp Physiol. 2011;301(5):R1467-78.

12. Freedman NS, Gazendam J, L L. abnormal sleep-wake cycles and the effect of environmental noise on sleep disruption in the intensive care unit. Am J Respir Crit Care Med. 2001;163(2):451-7.

13. Everson CA. Clinical assessment of blood leukocytes, serum cytokines, and serum immunoglobulins as responses to sleep deprivation in laboratory rats. Am J Physiol Regul Integr Comp Physiol. 2005;289(4):R1054-63.

14. Friese RS, Bruns $B$, Sinton CM. Sleep deprivation after septic insult increases mortality independent of age. J Trauma. 2009;66(1):50-4.

15. Zager A, Andersen ML, Ruiz FS, Antunes IB, Tufik S. Effects of acute and chronic sleep loss on immune modulation of rats. Am J Physiol Regul Integr Comp Physiol. 2007;293(1):R504-9.

16. Wang YH, Liang S, Xu DS, Lin X, He CY, Feng Y, et al. Effect and mechanism of senkyunolide I as an anti-migraine compound from Ligusticum chuanxiong. J Pharm Pharmacol. 2011;63(2):261-6. 
17. Peng $C$, Xie X, Wang L, Guo L, Hu T. Pharmacodynamic action and mechanism of volatile oil from Rhizoma Ligustici Chuanxiong Hort. on treating headache. Phytomedicine. 2009;16(1):25-34.

18. Ran X, Ma L, Peng C, Zhang H, Qin LP. Ligusticum chuanxiong Hort: a review of chemistry and pharmacology. Pharm Biol. 2011;49(11):1180-9.

19. Jianming Guo, Jin-AO Duan. Fast onset of action and the analgesic and sedative efficacy of essential oil from Rhizoma Chuanxiong after nasal administration. pharmazie. 2009;65:296-9.

20. Ji MH, Qiu LL, Tang H, Ju LS, Sun XR, Zhang H, et al. Sepsis-induced selective parvalbumin interneuron phenotype loss and cognitive impairments may be mediated by NADPH oxidase 2 activation in mice. J Neuroinflammation. 2015;12:182.

21. Hu Y, Duan M, Liang S, Wang Y, Feng Y. Senkyunolide I protects rat brain against focal cerebral ischemia-reperfusion injury by up-regulating p-Erk1/2, Nrf2/HO-1 and inhibiting caspase 3 . Brain Research. 2015;1605:39-48.

22. Wen $\mathrm{C}$, Xie T, K P. Acetate attenuates perioperative neurocognitive disorders in aged mice. Aging. 2020;12(4).

23. Liu SF, Malik AB. NF-kappa B activation as a pathological mechanism of septic shock and inflammation. Am J Physiol Lung Cell Mol Physiol. 2006;290(4):L622-L45.

24. Zhou RX, Li YY, Qu Y, Huang Q, Sun XM, Mu DZ, et al. Regulation of hippocampal neuronal apoptosis and autophagy in mice with sepsis-associated encephalopathy by immunity-related GTPase M1. CNS Neurosci Ther. 2020;26(2):177-88.

25. CA E. sustained sleep deprivation impairs host defense. Am J Physiol. 1993;265(5):R1148-54.

26. Ruan SS, Xiao YC, He PC, Wang Y, Ma T. Identification of Potential Gene Signatures Related to Sleep Deprivation. J Comput Biol. 2020;27(6):904-13.

27. Hu Y-y, Wang Y, Liang S, Yu X-I, Zhang L, Feng L-y, et al. Senkyunolide I attenuates oxygen-glucose deprivation/reoxygenation-induced inflammation in microglial cells. Brain Research. 2016;1649:12331.

28. Casey LC, Balk RA, RC B. Plasma cytokine and endotoxin levels correlate with survival in patients with the sepsis syndrome. Ann Intern Med. 1993;119:771-78.

29. Chaudhry N, Duggal AK. Sepsis Associated Encephalopathy. Adv Med. 2014;2014:762320.

30. Lemstra AW, Groen in't Woud JC, Hoozemans JJ, van Haastert ES, Rozemuller AJ, Eikelenboom P, et al. Microglia activation in sepsis: a case-control study. J Neuroinflammation. 2007;4:4.

31. Henry CJ, Huang Y, Wynne AM, Godbout JP. Peripheral lipopolysaccharide (LPS) challenge promotes microglial hyperactivity in aged mice that is associated with exaggerated induction of both proinflammatory IL-1 beta and anti-inflammatory IL-10 cytokines. Brain Behav Immun. 2009;23(3):30917.

32. M. Michels, L. G. Danielski F. Neuroinflammation『microglial activation during sepsis. Current Neurovascular Research. 2014;11(3):263-70. 
33. Wadhwa M, Chauhan G, Roy K, Sahu S, Deep S, Jain V, et al. Caffeine and Modafinil Ameliorate the Neuroinflammation and Anxious Behavior in Rats during Sleep Deprivation by Inhibiting the Microglia Activation. Front Cell Neurosci. 2018;12:49.

\section{Figures}
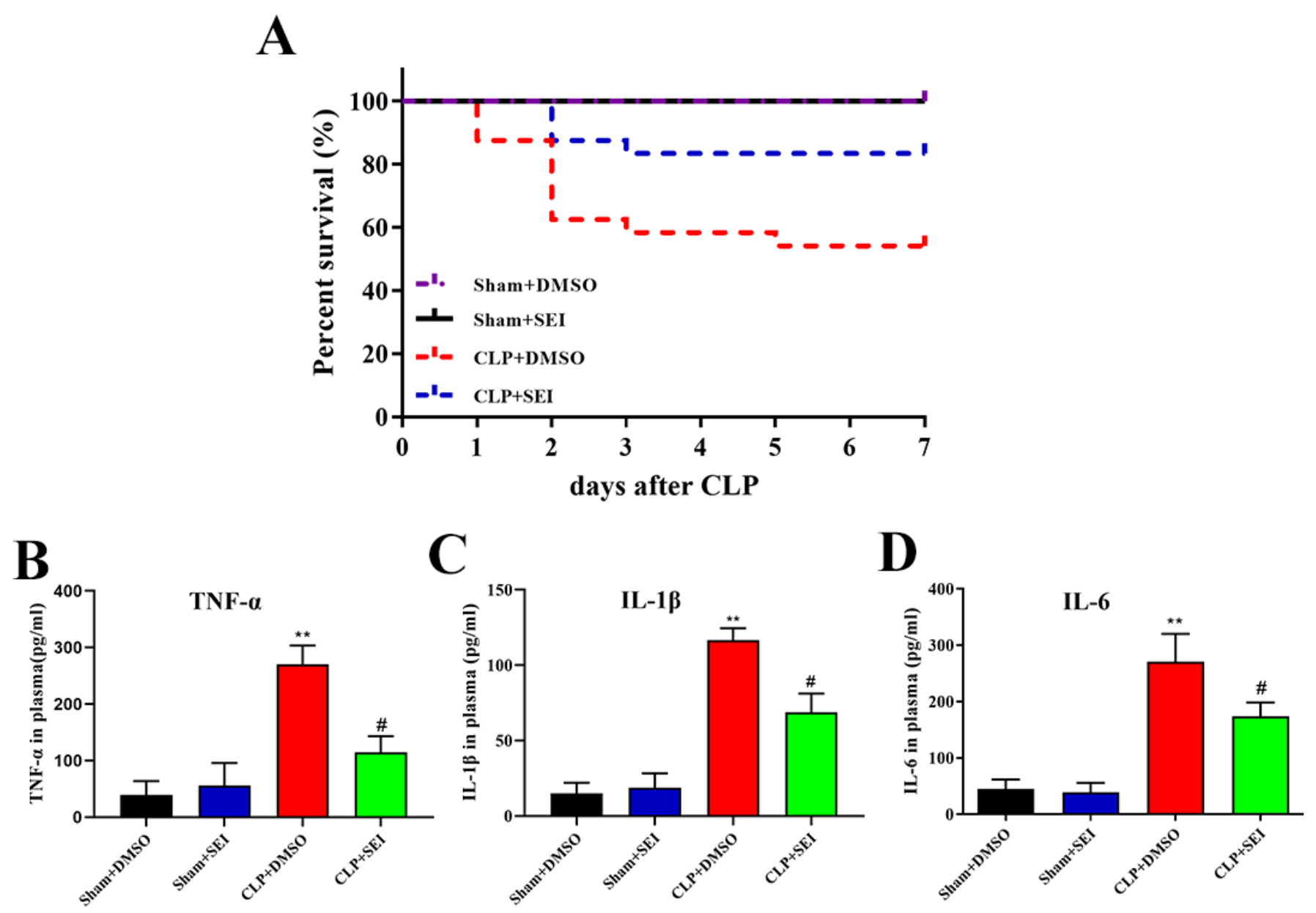

Figure 1

Senkyunolide I treatment improved survival rate and reduced systemic inflammation of septic mice. (A)Survival analysis of CLP mice ( $n=24$ for each group, $p=0.0048$ for CLP+SEl vs. CLP+DMSO). (B-D) Plasma levels of TNF-a (B), IL-1 $\beta$ (C) and IL-6 (D). Data were displayed as the mean \pm SEM ( $n=6$ for each group). ${ }^{\star *} P<0.01$ vs. Sham groups, $\# P<0.05$ vs. CLP+DMSO group. SEl: Senkyunolide I. CLP: cecal ligation and puncture. 
A
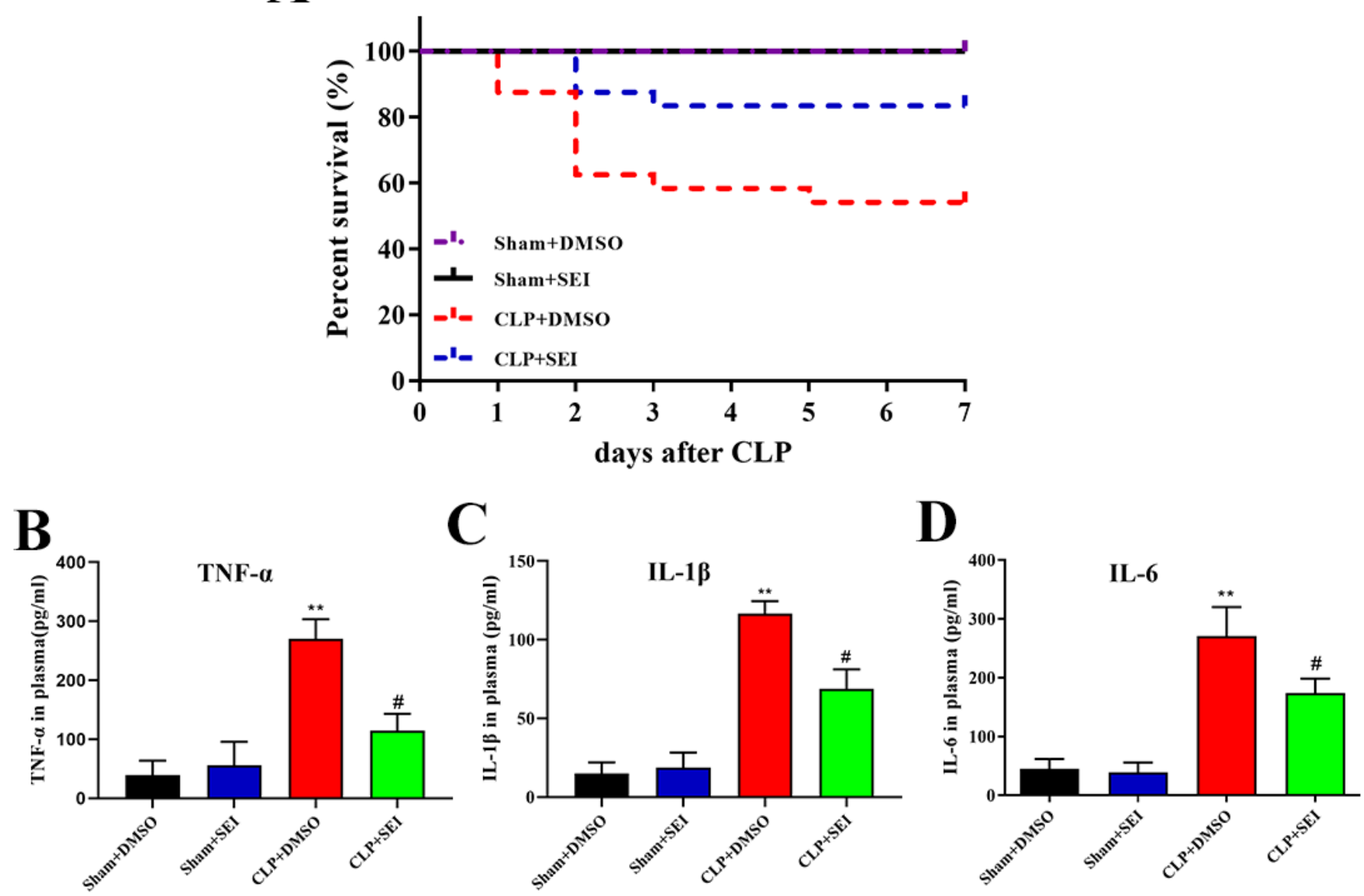

Figure 1

Senkyunolide I treatment improved survival rate and reduced systemic inflammation of septic mice. (A)Survival analysis of CLP mice ( $n=24$ for each group, $p=0.0048$ for CLP+SEl vs. CLP+DMSO). (B-D) Plasma levels of TNF- $a(B), I L-1 \beta(C)$ and IL-6 (D). Data were displayed as the mean \pm SEM ( $n=6$ for each group). ${ }^{\star} \mathrm{P}<0.01$ vs. Sham groups, $\# \mathrm{P}<0.05$ vs. CLP+DMSO group. SEl: Senkyunolide I. CLP: cecal ligation and puncture. 


\section{A}

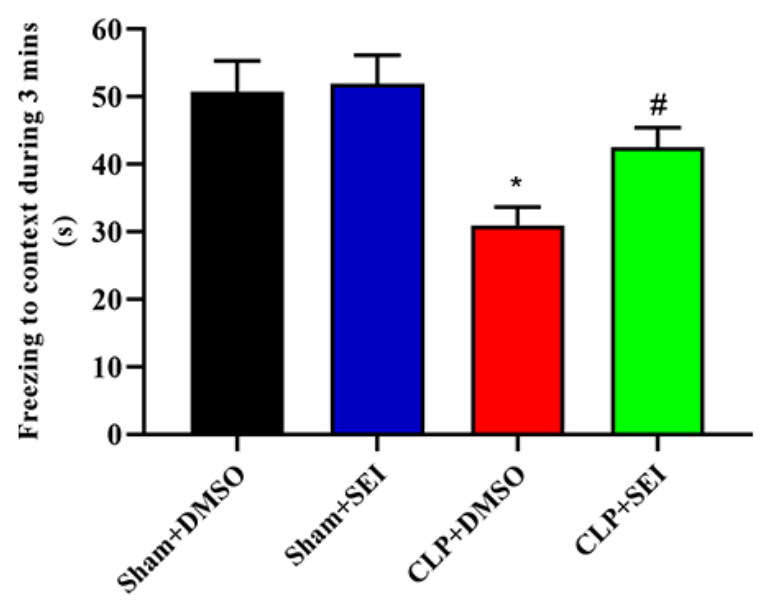

B

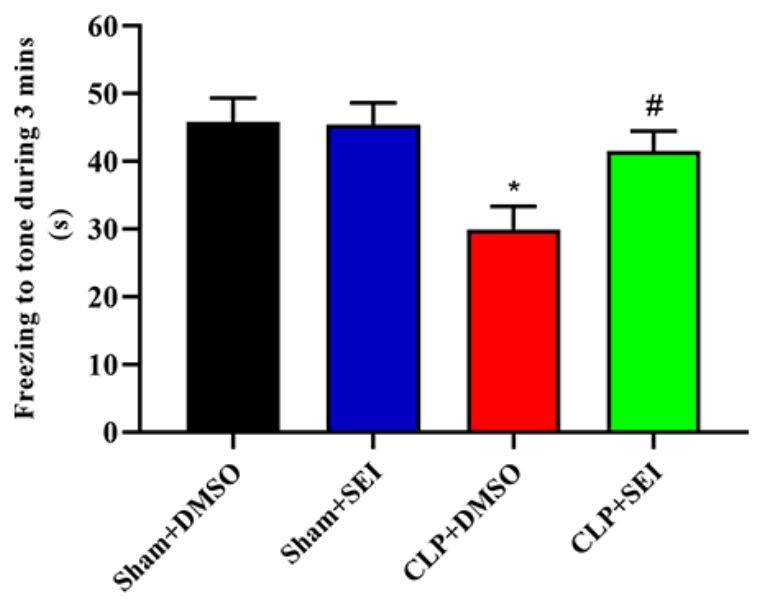

Figure 2

Senkyunolide I treatment protected against cognitive dysfunction in septic mice. (A) Freezing to context and $(B)$ freezing to tone were tested at the $24 \mathrm{~h}$ after surgery, respectively. Data were shown as mean \pm SEM ( $n=8$ for each group). ${ }^{*} P<0.05$ vs. sham groups. \#P $<0.05$ vs. CLP+DMSO group. SEl: Senkyunolide I. CLP: cecal ligation and puncture.

A

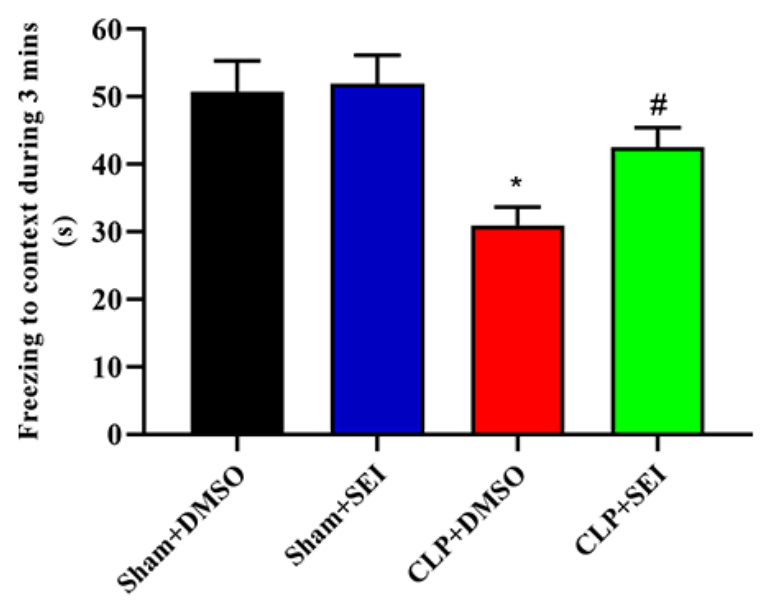

B

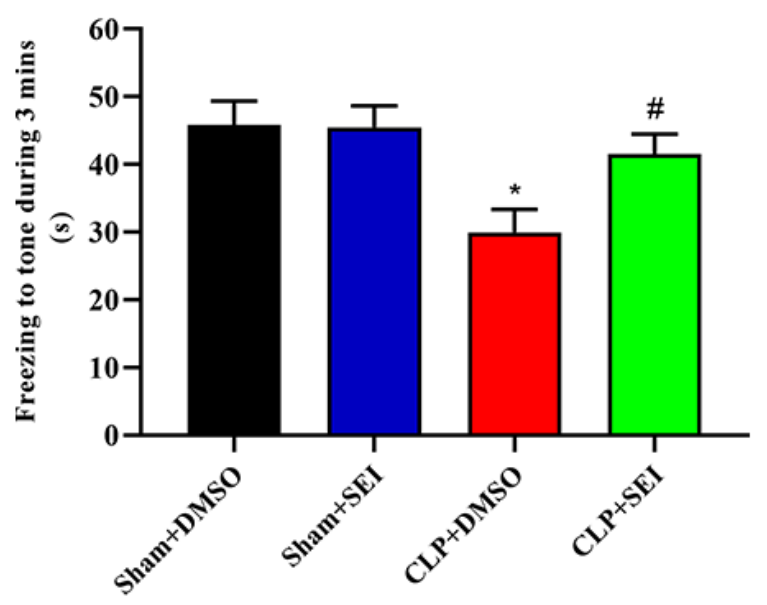

Figure 2

Senkyunolide I treatment protected against cognitive dysfunction in septic mice. (A) Freezing to context and (B) freezing to tone were tested at the $24 \mathrm{~h}$ after surgery, respectively. Data were shown as mean \pm SEM ( $n=8$ for each group). ${ }^{\star} P<0.05$ vs. sham groups. \#P $<0.05$ vs. CLP+DMSO group. SEl: Senkyunolide I. CLP: cecal ligation and puncture. 


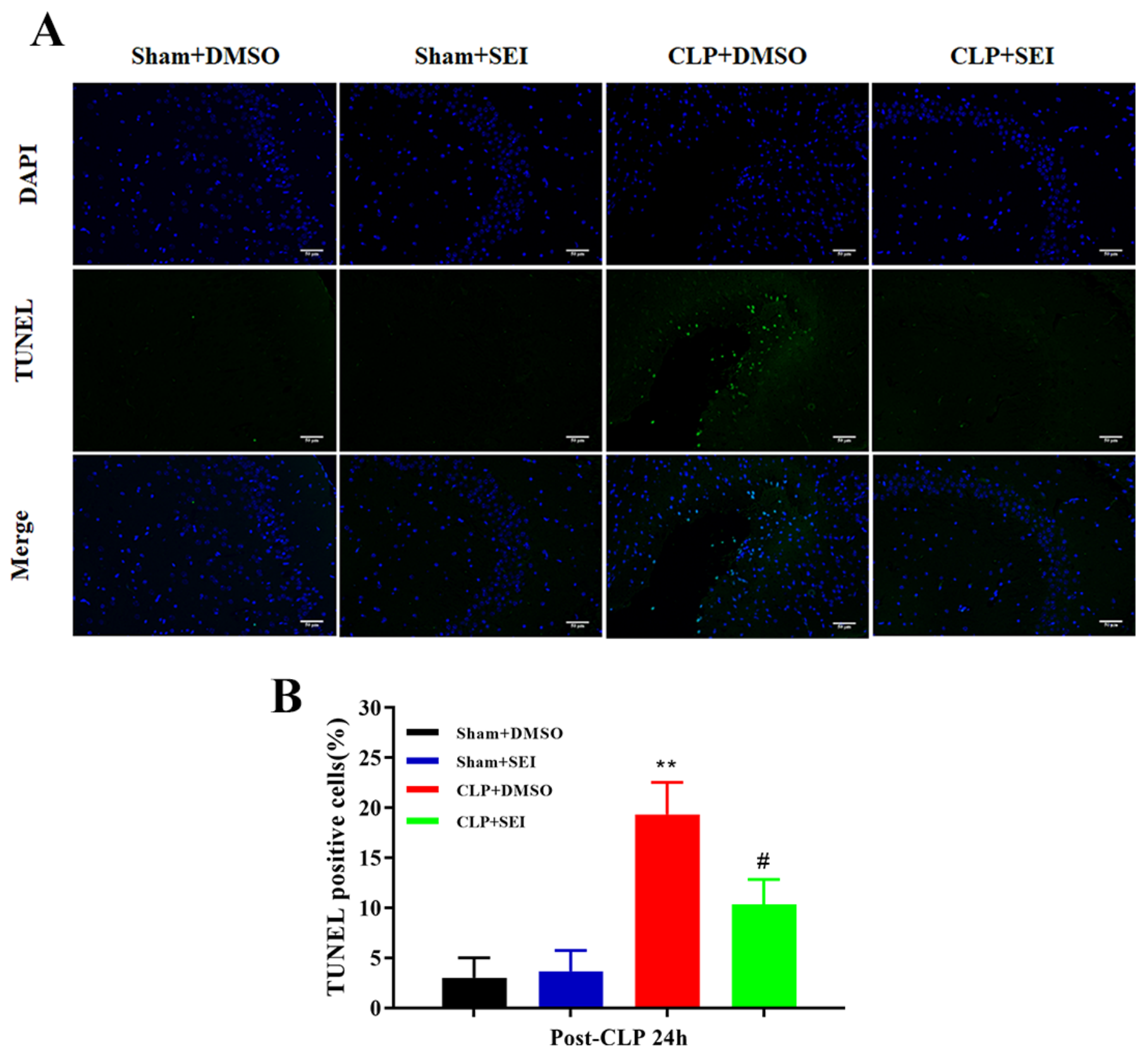

Figure 3

Senkyunolide I administration significantly reduced the number of apoptotic cells in the hippocampus of septic mice. (A) Representative images of TUNEL fluorescence of coronal sections of the hippocampus at 24h after CLP surgery. (B) Quantification of TUNEL-positive cells. Values were shown as mean \pm SEM ( $n=6$ for each group). ${ }^{\star \star P}<0.01$ vs. Sham groups; $\# P<0.05$ vs. CLP group. SEl: Senkyunolide I. CLP: cecal ligation and puncture. Scale bar $=50 \mu \mathrm{m}$. 


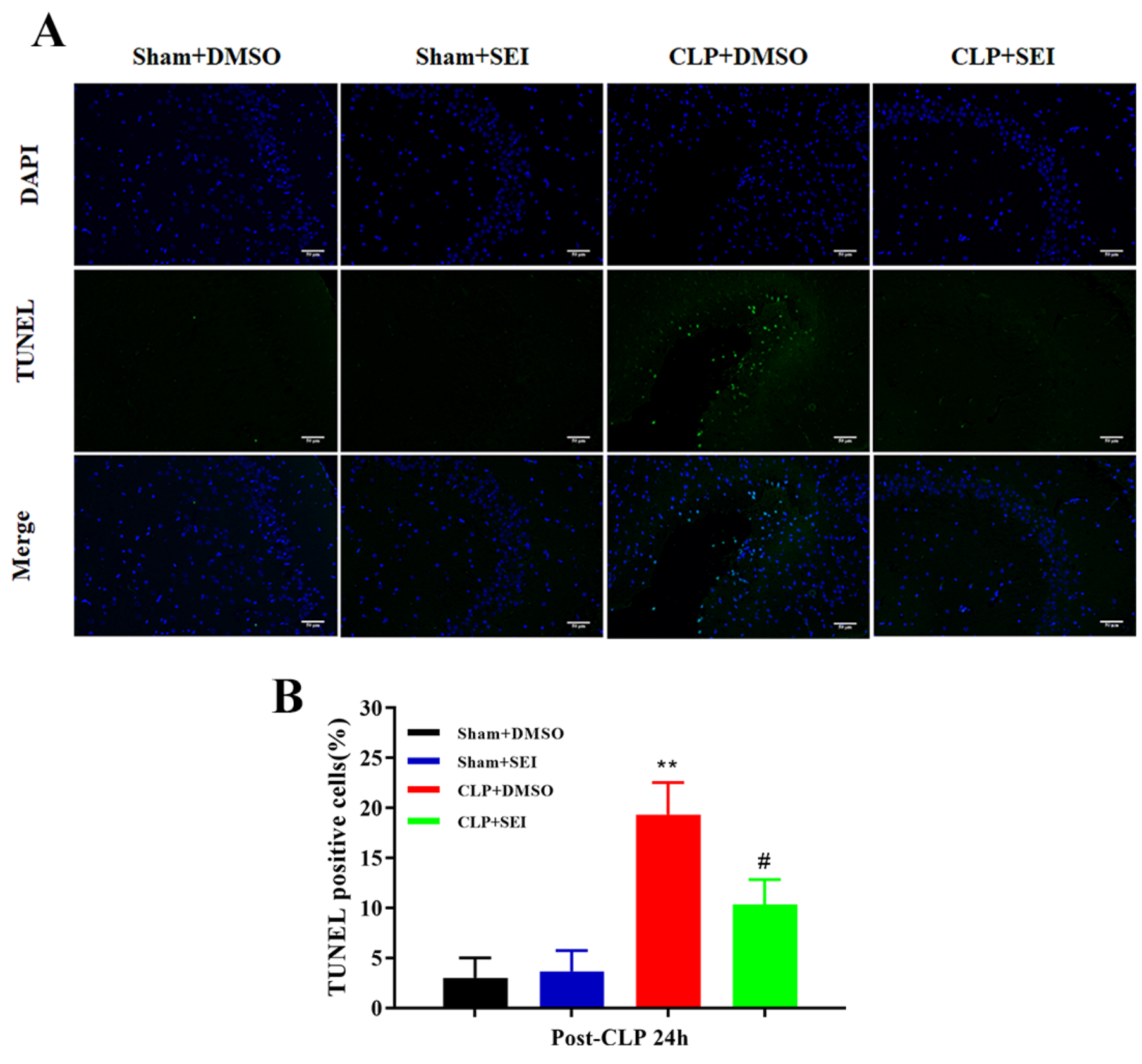

Figure 3

Senkyunolide I administration significantly reduced the number of apoptotic cells in the hippocampus of septic mice. (A) Representative images of TUNEL fluorescence of coronal sections of the hippocampus at 24h after CLP surgery. (B) Quantification of TUNEL-positive cells. Values were shown as mean \pm SEM ( $n=6$ for each group). ${ }^{\star \star P}<0.01$ vs. Sham groups; $\# P<0.05$ vs. CLP group. SEl: Senkyunolide I. CLP: cecal ligation and puncture. Scale bar $=50 \mu \mathrm{m}$. 


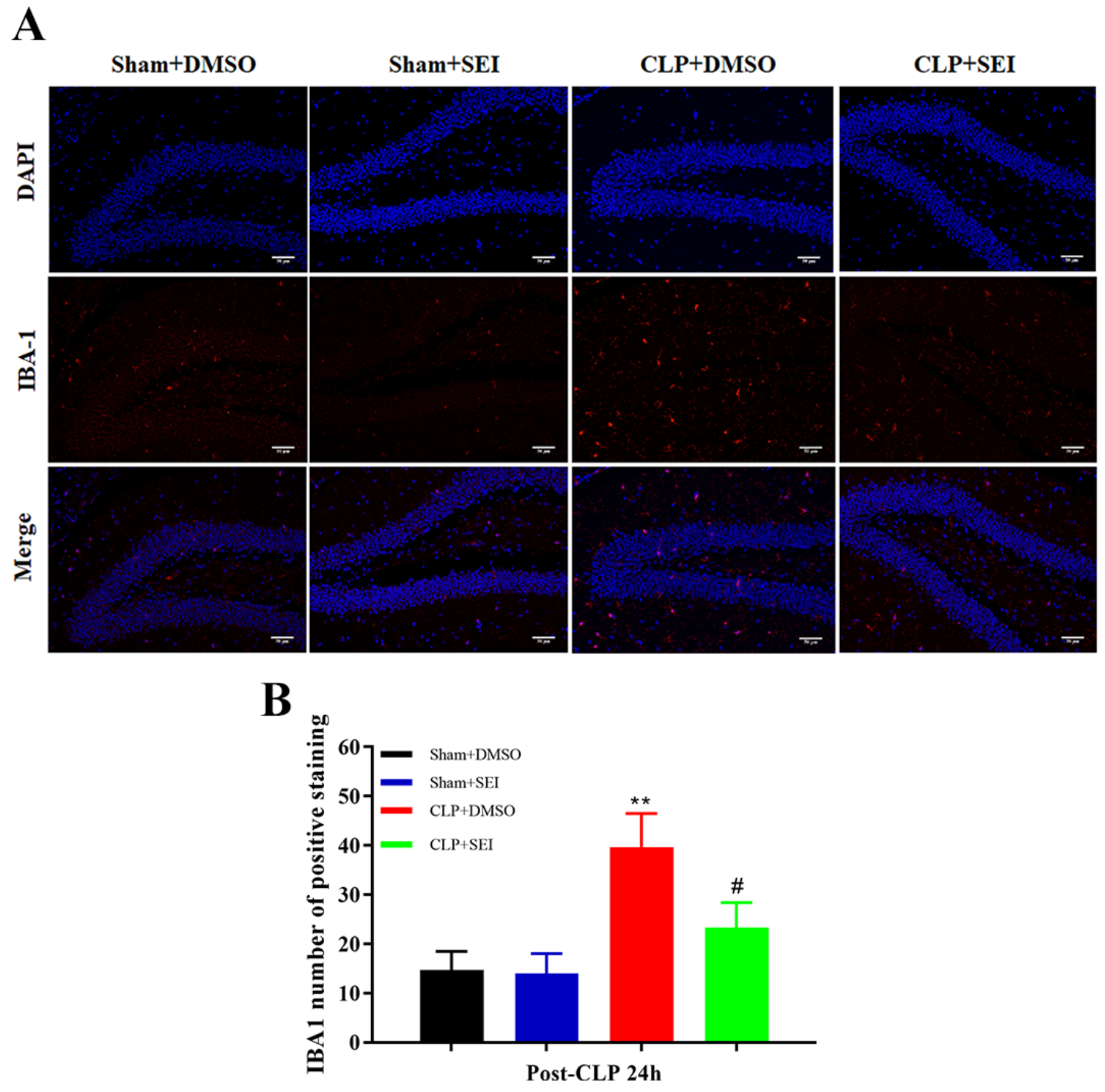

Figure 4

Sepsis-induced IBA-1 activation in the hippocampus were attenuated by Senkyunolide I treatment. (A) Representative images of IBA-1 expression at $24 \mathrm{~h}$ after CLP surgery. (B) Bar graphs indicated the number of IBA-1-labeled microglia in the four groups. Data were presented as mean \pm SEM ( $n=6$ for each group). **P<0.01 vs. sham groups, \#P<0.05 vs. CLP group. SEl: Senkyunolide I. CLP: cecal ligation and puncture. Scale bar $=50 \mu \mathrm{m}$. 


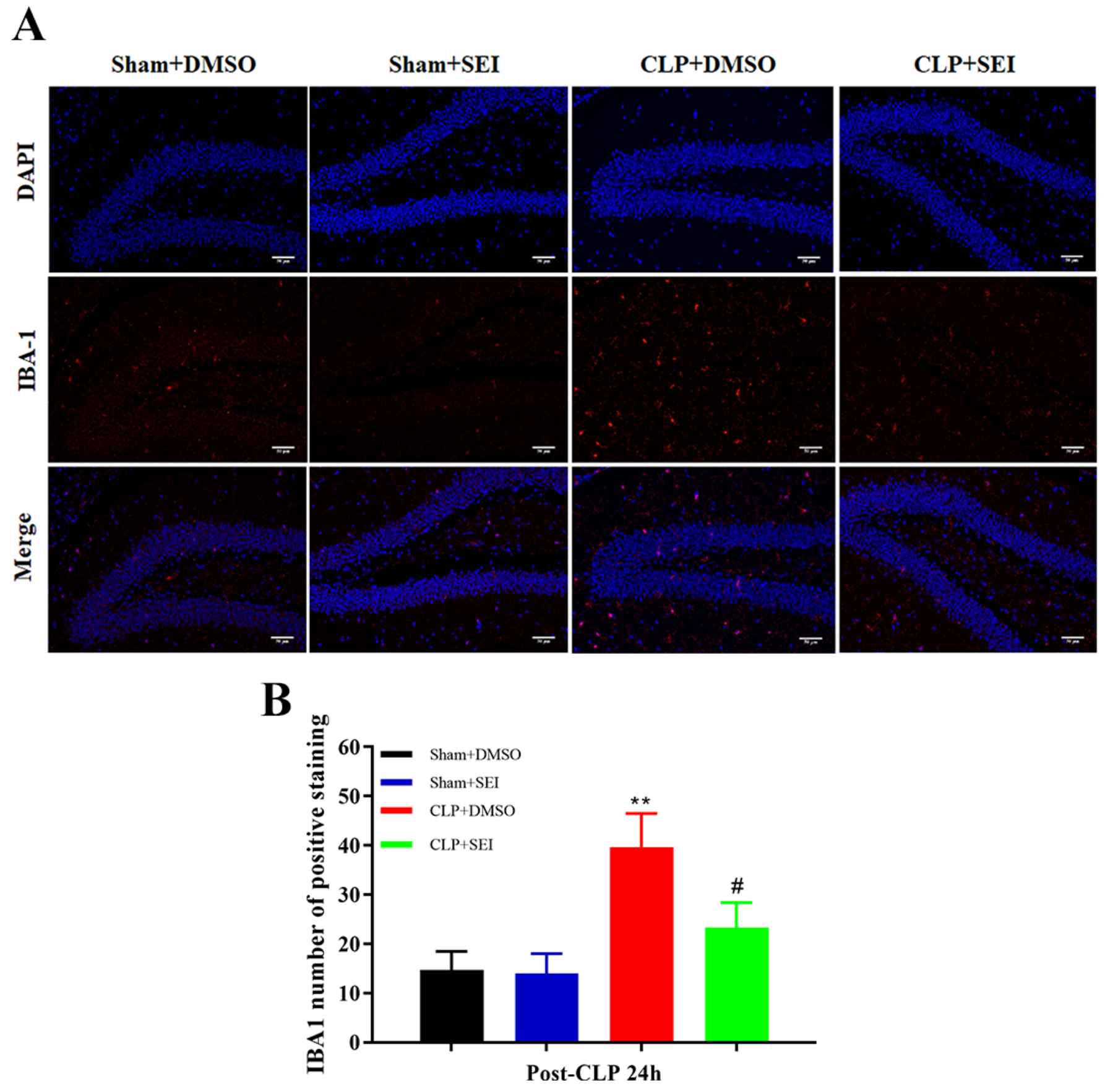

Figure 4

Sepsis-induced IBA-1 activation in the hippocampus were attenuated by Senkyunolide I treatment. (A) Representative images of IBA-1 expression at $24 \mathrm{~h}$ after CLP surgery. (B) Bar graphs indicated the number of IBA-1-labeled microglia in the four groups. Data were presented as mean \pm SEM ( $n=6$ for each group). **P<0.01 vs. sham groups, \#P<0.05 vs. CLP group. SEl: Senkyunolide I. CLP: cecal ligation and puncture. Scale bar $=50 \mu \mathrm{m}$. 

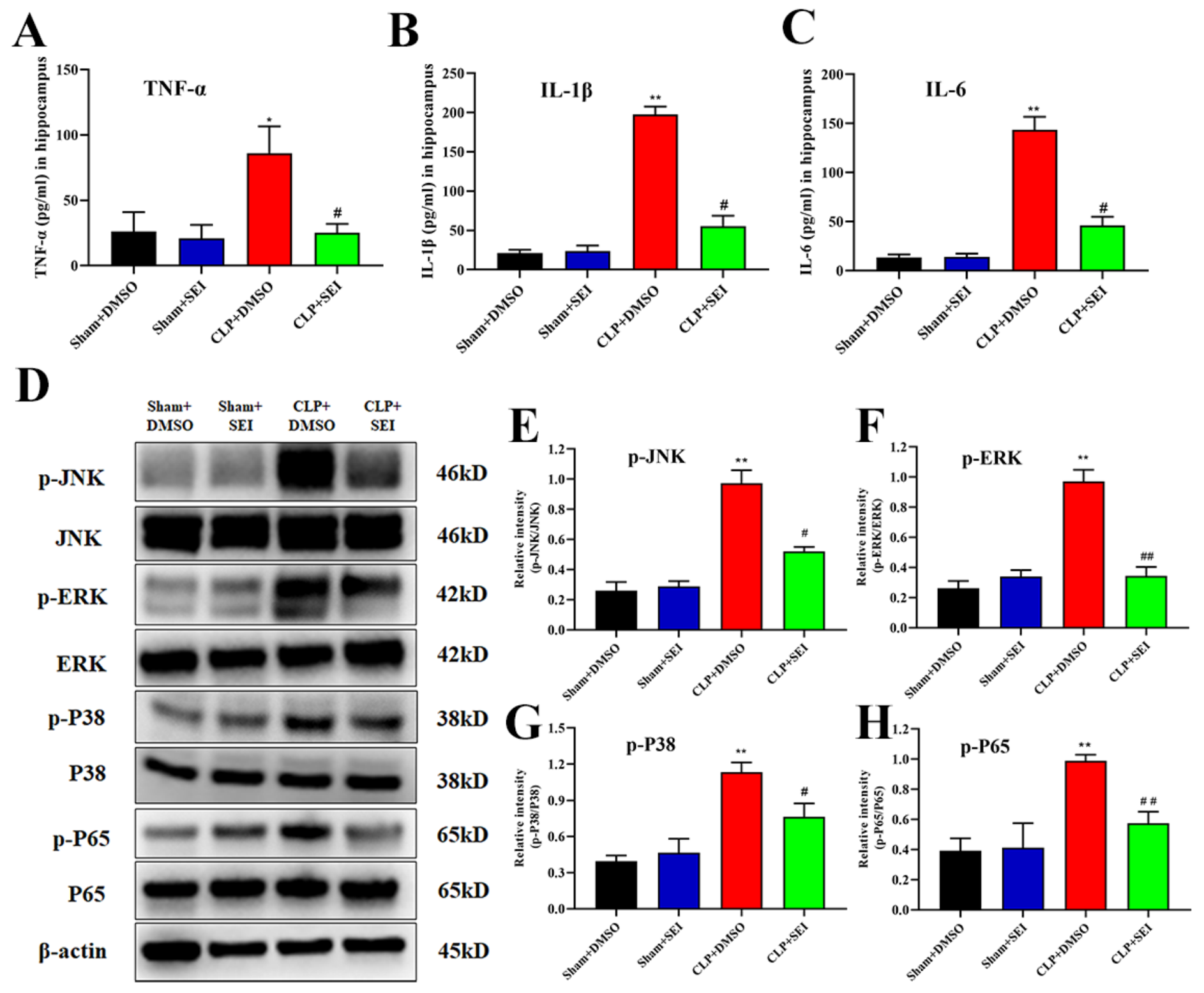

Figure 5

Sepsis-induced neuroinflammation was attenuated by Senkyunolide I treatment. (A) TNF-a, (B) IL-1 $\beta$ and (C) IL-6 in the hippocampus homogenate were measured at 24h after CLP surgery. (D) Representative bands showed the levels of p-JNK, JNK, p-ERK, ERK, p-P38, P38, p-P65 and P65 protein in hippocampus tissue at $24 \mathrm{~h}$ after surgery. The relative quantification of p-JNK/JNK (E), p-ERK/ERK (F), p-P38/P38 (G), p$P 65 / P 65(H)$ were analyzed. Values were mean \pm SEM ( $n=6$ for each group). ${ }^{*} P<0.01$ vs. sham groups; $\# \# P<0.01$ vs. CLP+DMSO group; \#P < 0.05 vs. CLP+DMSO group. SEl: Senkyunolide I. CLP: cecal ligation and puncture. 

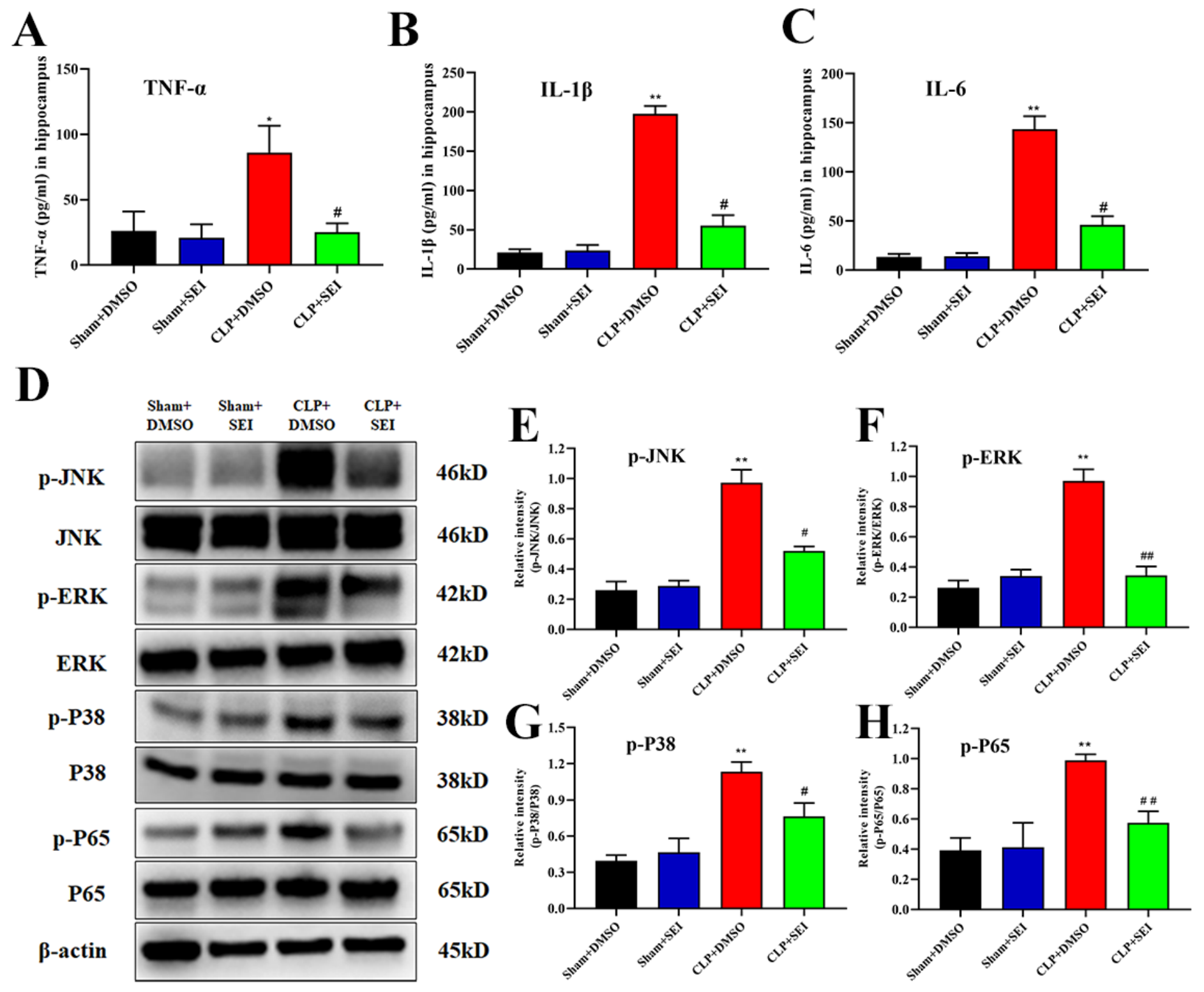

Figure 5

Sepsis-induced neuroinflammation was attenuated by Senkyunolide I treatment. (A) TNF-a, (B) IL-1 $\beta$ and (C) IL-6 in the hippocampus homogenate were measured at 24h after CLP surgery. (D) Representative bands showed the levels of p-JNK, JNK, p-ERK, ERK, p-P38, P38, p-P65 and P65 protein in hippocampus tissue at $24 \mathrm{~h}$ after surgery. The relative quantification of p-JNK/JNK (E), p-ERK/ERK (F), p-P38/P38 (G), p$P 65 / P 65(H)$ were analyzed. Values were mean \pm SEM ( $n=6$ for each group). ${ }^{*} P<0.01$ vs. sham groups; $\# \# P<0.01$ vs. CLP+DMSO group; \#P < 0.05 vs. CLP+DMSO group. SEl: Senkyunolide I. CLP: cecal ligation and puncture. 
A hippocampus
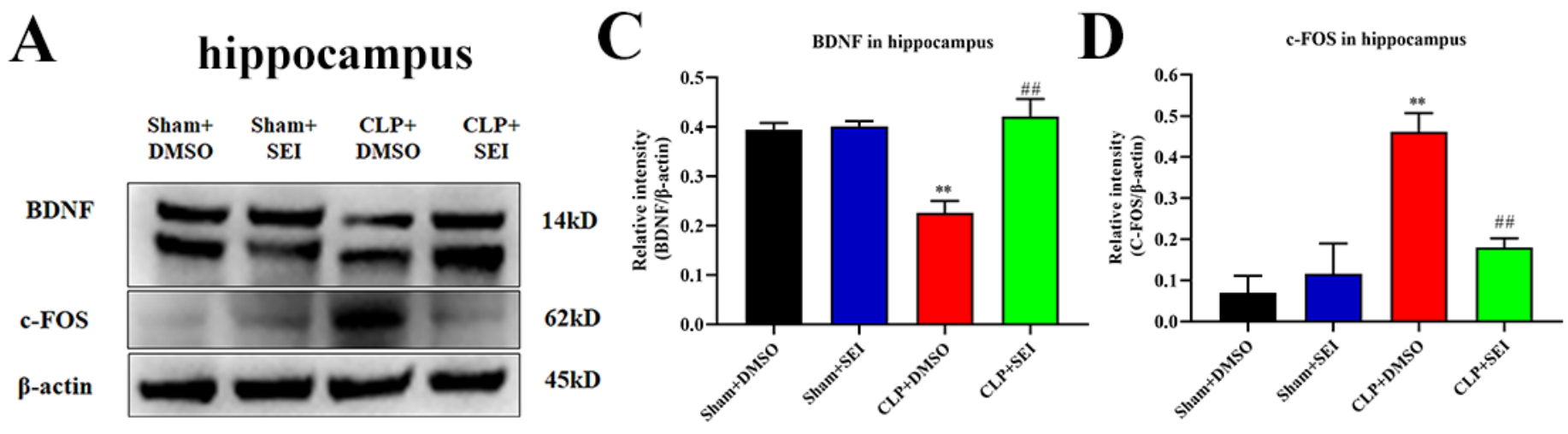

\section{B}

\section{cortex}
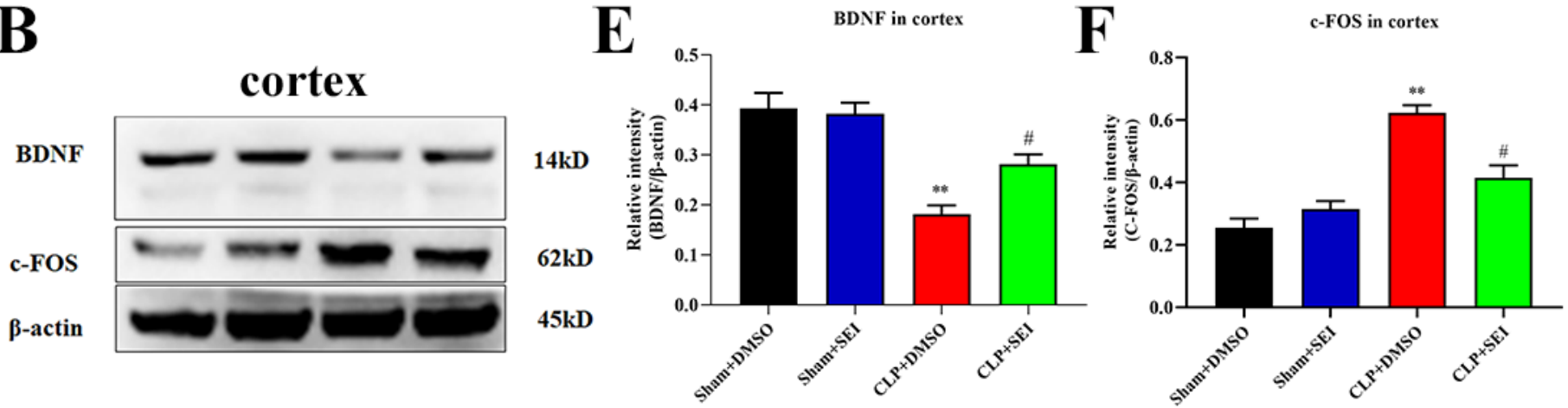

Figure 6

Sleep deprivation of septic mice was attenuated by Senkyunolide I treatment. (A-B) Sleep deprivation markers BDNF and c-FOS in both hippocampus and cerebral cortex were measured by western blot assay. (C-F) The relative quantification of BDNF/ $\beta$-actin and $\mathrm{c}-\mathrm{FOS} / \beta$-actin in both hippocampus and cerebral cortex were analyzed. Values were presented as mean \pm SEM ( $n=6$ for each group). ${ }^{*} P<0.01$ vs. sham groups; \#\#P< 0.01 vs. CLP+DMSO group; \#P<0.05 vs. CLP+DMSO group. SEl: Senkyunolide I. CLP: cecal ligation and puncture. 
A hippocampus
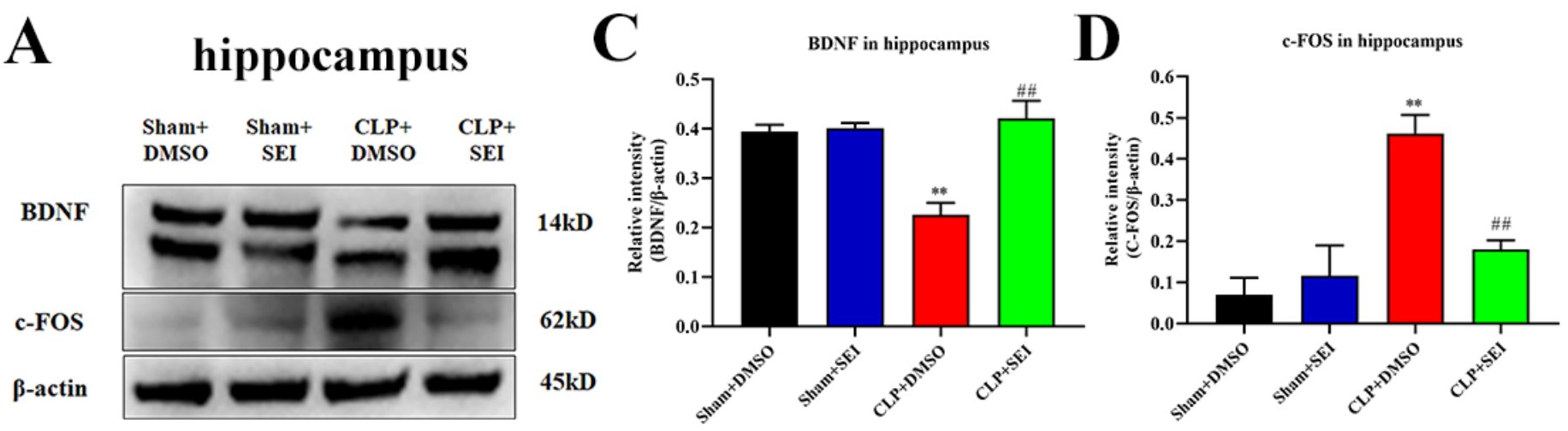

B
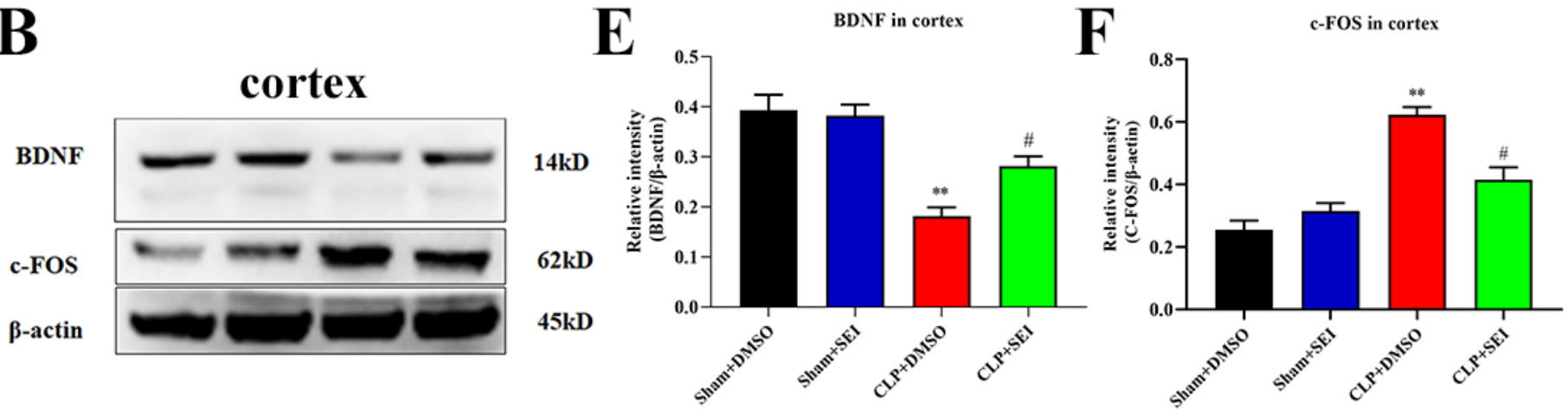

Figure 6

Sleep deprivation of septic mice was attenuated by Senkyunolide I treatment. (A-B) Sleep deprivation markers BDNF and c-FOS in both hippocampus and cerebral cortex were measured by western blot assay. (C-F) The relative quantification of BDNF/ $\beta$-actin and $\mathrm{c}-\mathrm{FOS} / \beta$-actin in both hippocampus and cerebral cortex were analyzed. Values were presented as mean \pm SEM ( $n=6$ for each group). ${ }^{\star *} P<0.01$ vs. sham groups; \#\#P< 0.01 vs. CLP+DMSO group; \#P<0.05 vs. CLP+DMSO group. SEl: Senkyunolide I. CLP: cecal ligation and puncture. 
A
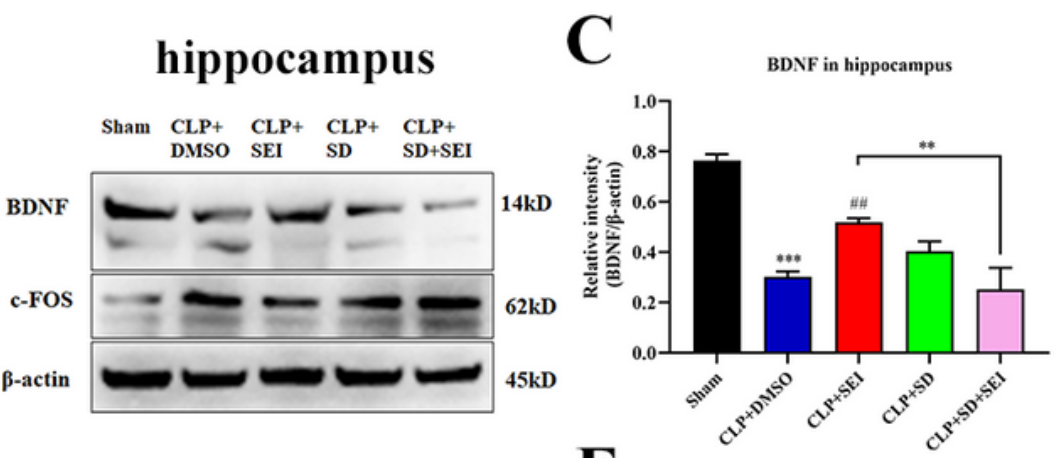

B
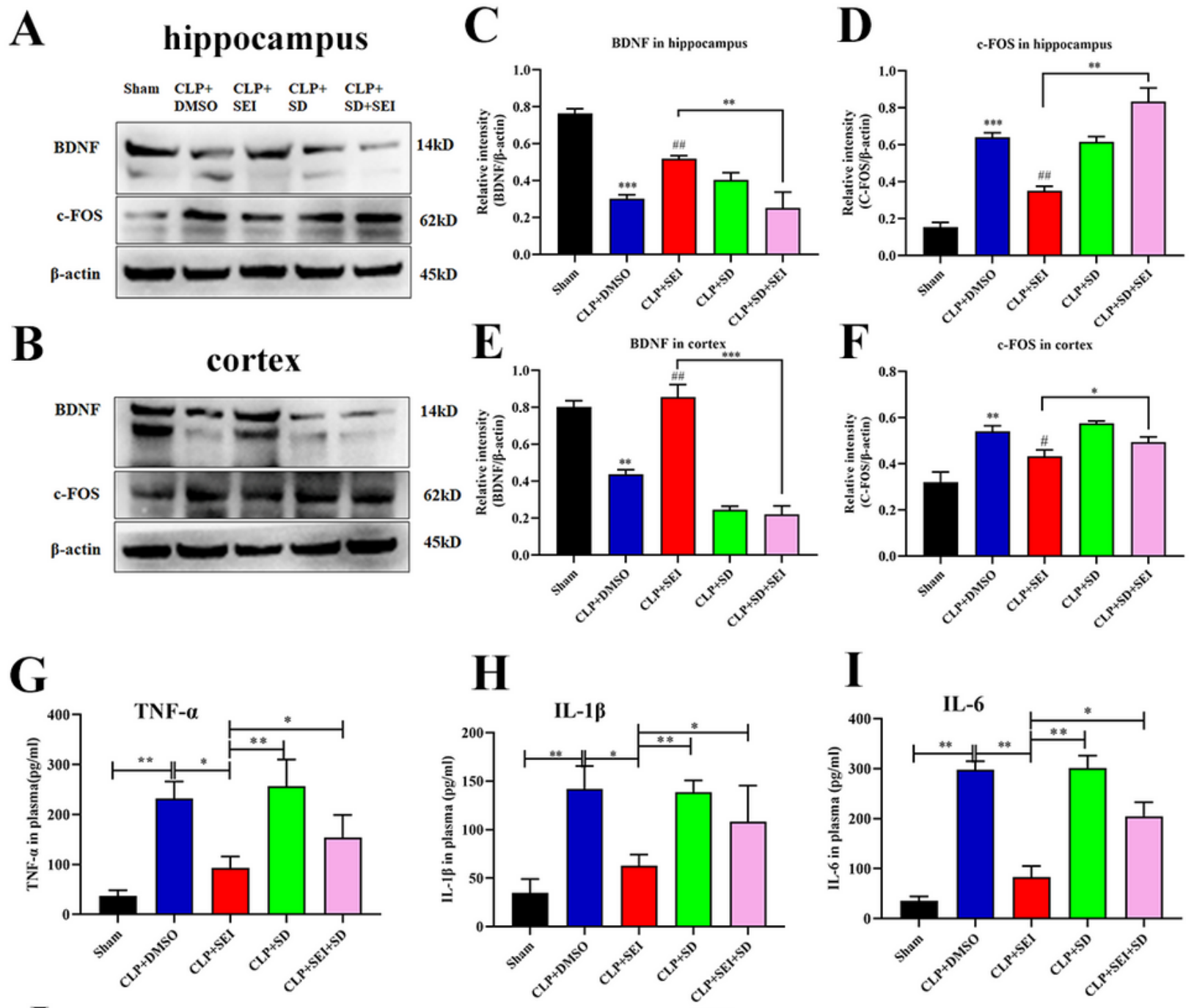

$\mathbf{J}$

K
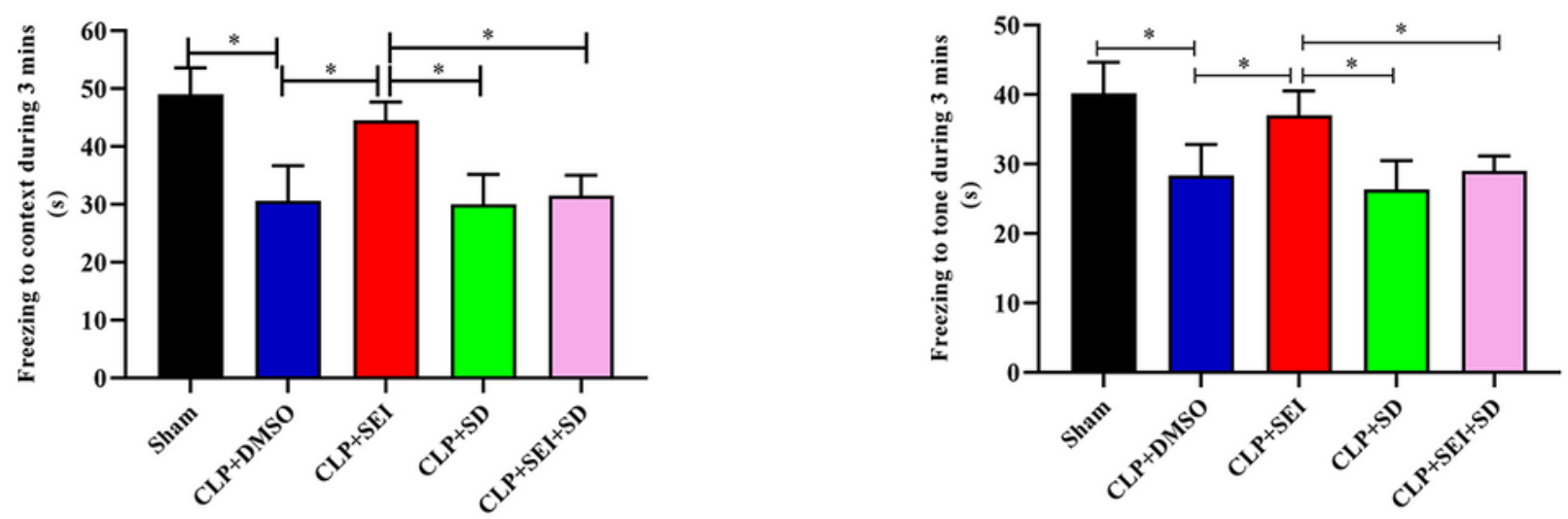

Figure 7

Improvement of cognitive dysfunction by Senkyunolide I of septic mice was reversed by sleep deprivation. Sleep deprivation was induced for $24 \mathrm{~h}$ after surgery. (A-B) Expression levels of BDNF and cFOS were detected by Western blot. (C-F) The relative quantification of BDNF/ $\beta$-actin and c-FOS/ $\beta$-actin in both hippocampus and cerebral cortex were analyzed. The levels of TNF- $\alpha(G), I L-1 \beta(H)$ and IL- $6(I)$ in the serum were analyzed by ELISA. Freezing to context $(\mathrm{J})$ and freezing to tone $(\mathrm{K})$ were tested at $24 \mathrm{~h}$ after 
surgery, respectively. Values were presented as mean \pm SEM ( $n=6-8$ for each group). ${ }^{\star *} P<0.01$ vs. sham groups; ${ }^{P}<0.05$ vs. sham groups; \#\#P< 0.01 vs. CLP+DMSO group; \#P<0.05 vs. CLP+DMSO group. SEl: Senkyunolide I. CLP: cecal ligation and puncture.

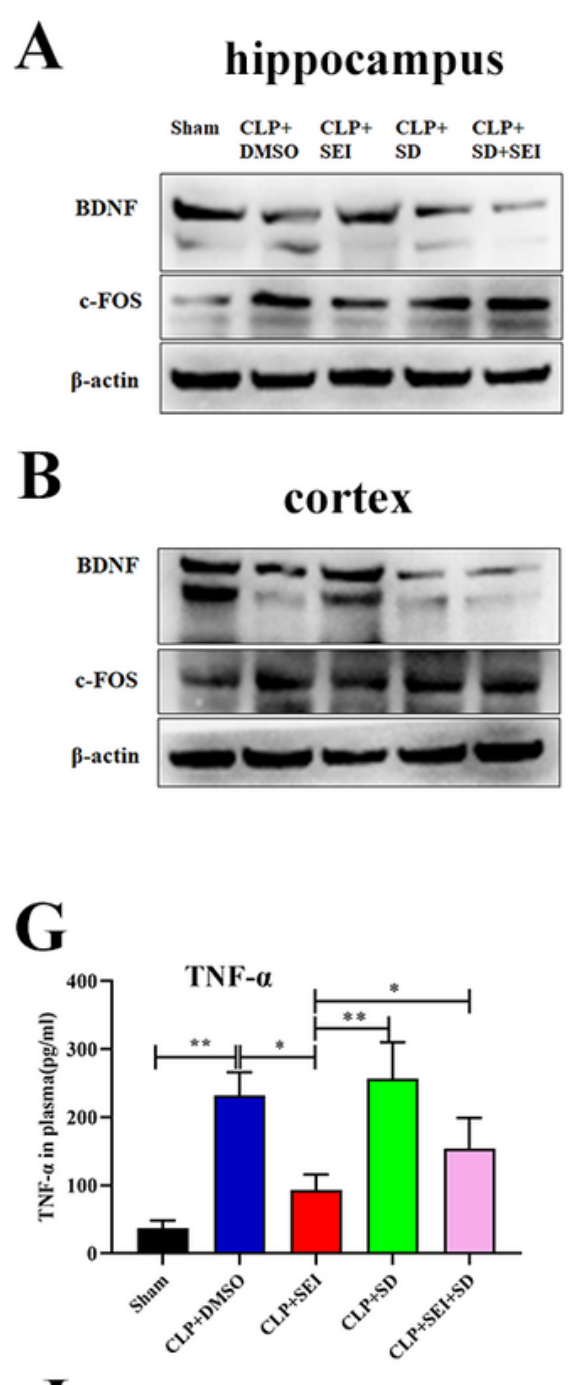

$\mathbf{J}$

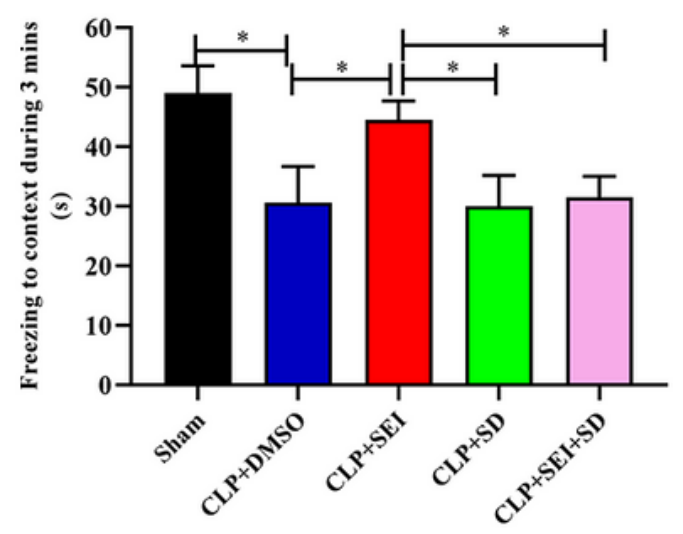

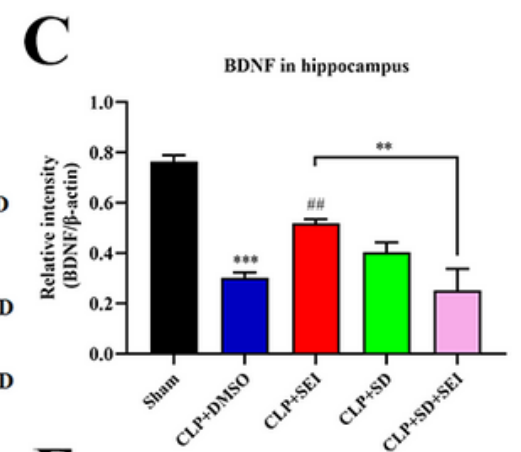

$\mathbf{E}$
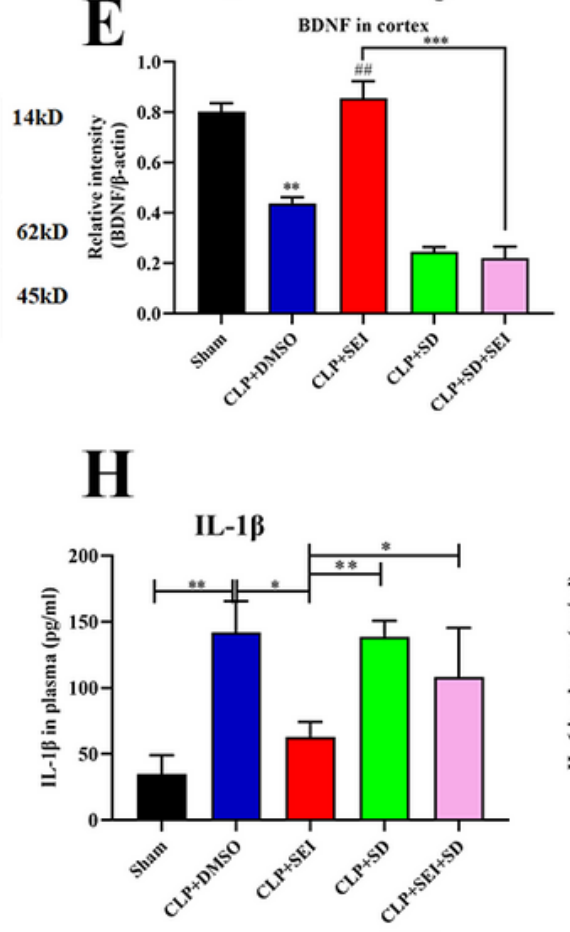

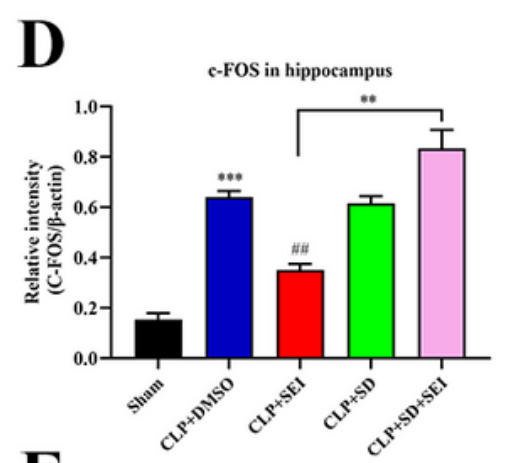

$\mathbf{F}$

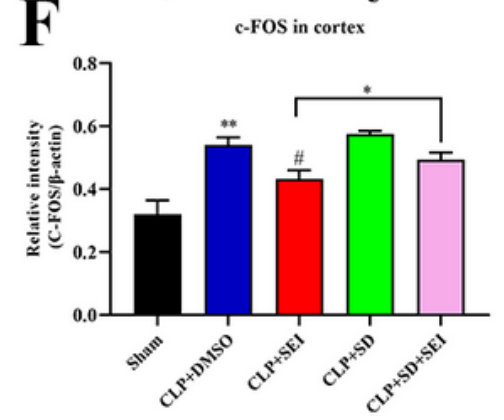

I

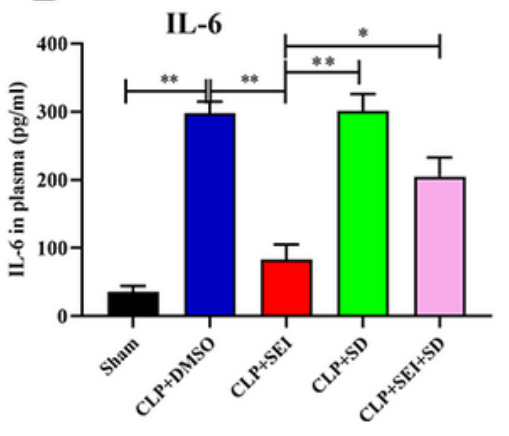

K

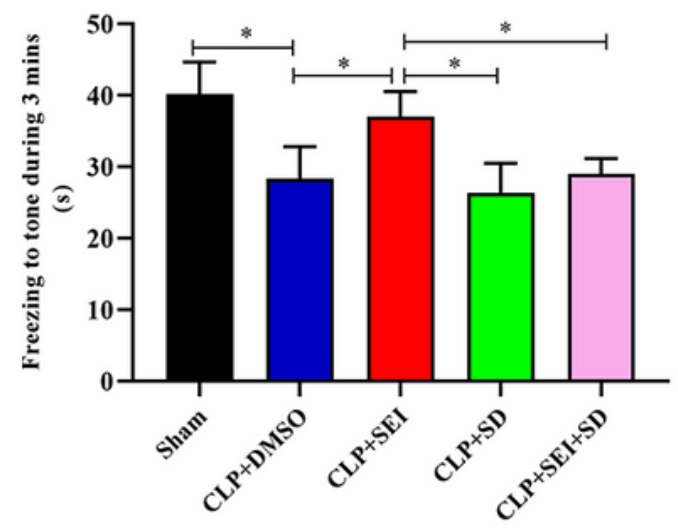

Figure 7

Improvement of cognitive dysfunction by Senkyunolide I of septic mice was reversed by sleep deprivation. Sleep deprivation was induced for 24h after surgery. (A-B) Expression levels of BDNF and c- 
FOS were detected by Western blot. (C-F) The relative quantification of BDNF/ $\beta$-actin and c-FOS/ $\beta$-actin in both hippocampus and cerebral cortex were analyzed. The levels of TNF-a (G), IL-1 $\beta(H)$ and IL-6 $(\mathrm{I})$ in the serum were analyzed by ELISA. Freezing to context $(\mathrm{J})$ and freezing to tone $(\mathrm{K})$ were tested at $24 \mathrm{~h}$ after surgery, respectively. Values were presented as mean \pm SEM ( $n=6-8$ for each group). ${ }^{\star *} P<0.01$ vs. sham groups; $* P<0.05$ vs. sham groups; \#\#P<0.01 vs. CLP+DMSO group; \#P<0.05 vs. CLP+DMSO group. SEl: Senkyunolide I. CLP: cecal ligation and puncture. 